\title{
Partial sums of analytic functions of bounded turning with applications
}

\author{
MASLINA DARUS* and RABHA W. IBRAHIM \\ School of Mathematical Sciences, Faculty of Science and Technology \\ Universiti Kebangsaan Malaysia, Bangi 43600, Selangor Darul Ehsan, Malaysia \\ E-mail: maslina@ukm.my/rabhaibrahim@yahoo.com
}

\begin{abstract}
In this article, we determine certain conditions under which the partial sums of the multiplier integral operators of analytic univalent functions of bounded turning are also of bounded turning.
\end{abstract}

Mathematical subject classification: $30 \mathrm{C} 45$.

Key words: multiplier integral operator, partial sums, bounded turning.

\section{Introduction}

Let $\mathcal{H}$ be the class of functions analytic in the open unit disk $U=\{z: z \in$ $\mathbb{C}$ and $|z|<1\}$ and $\mathcal{H}[a, n]$ be the subclass of $\mathcal{H}$ consisting of functions of the form

$$
f(z)=a+a_{n} z^{n}+a_{n+1} z^{n+1}+\cdots .
$$

Let $\mathcal{A}$ be the subclass of $\mathcal{H}$ consisting of functions of the form

$$
f(z)=z+\sum_{n=2}^{\infty} a_{n} z^{n}, \quad(z \in U) .
$$

For $0 \leq \mu<1$, let $B(\mu)$ denote the class of functions $f$ of the form (1) so that $\Re\left\{f^{\prime}\right\}>\mu \in U$. The functions in $B(\mu)$ are called functions of bounded turning

\#CAM-79/09. Received: 15/III/09. Accepted: 15/III/09.

*Corresponding author. 
(c.f. [1, Vol. II]). By the Nashiro-Warschowski Theorem (see e.g. [1, Vol. I]) the functions in $B(\mu)$ are univalent and also close-to-convex in $U$.

For $f$ of the form (1), several interesting families of integral operators, which have been investigated rather extensively in analytic function theory, including each of the following integral operators (see [2-10]),

$$
\begin{aligned}
P_{b}^{a} f(z)= & \frac{(b+1)^{a}}{z^{b} \Gamma(a)} \int_{0}^{z} t^{b-1}\left(\log \frac{z}{t}\right)^{a-1} f(t) d t \\
= & z+\sum_{n=2}^{\infty}\left(\frac{b+1}{b+n}\right)^{a} a_{n} z^{n}, \quad(z \in U) \\
& (a>0, \quad b>-1, \quad z \in U, \quad f \in \mathcal{A})
\end{aligned}
$$

and

$$
\begin{aligned}
J_{c} f(z)= & \frac{c+1}{z^{c}} \int_{0}^{z} t^{c-1} f(t) d t \\
= & z+\sum_{n=2}^{\infty}\left(\frac{c+1}{c+n}\right) a_{n} z^{n}, \quad(z \in U) \\
& (c>-1, \quad z \in U, \quad f \in \mathcal{A}) .
\end{aligned}
$$

Also, we define a general integral operator as the following:

$$
\begin{gathered}
J_{\lambda, \delta}^{k} f(z)=z+\sum_{n=2}^{\infty} \frac{a_{n}}{[1+(n-1) \lambda]^{k} C(\delta, n)} z^{n}, \\
\left(k \in \mathbb{N}_{0}, \quad \lambda \geq 0, \quad \delta \geq 0 \quad z \in U, \quad f \in \mathcal{A}\right),
\end{gathered}
$$

where

$$
C(\delta, n)=\left(\begin{array}{c}
n+\delta-1 \\
\delta
\end{array}\right)=\frac{\Gamma(n+\delta)}{\Gamma(n) \Gamma(\delta+1)} .
$$

Remark 1.1. When $\lambda=0$, operator (4) gives Noor integral operator (see $[11,12])$.

The $m$-th partial sums of the operators (2-4) are respectively given by

$$
P_{m}(z)=z+\sum_{n=2}^{m}\left(\frac{b+1}{b+n}\right)^{a} a_{n} z^{n}, \quad(z \in U),
$$




$$
J_{m}(z)=z+\sum_{n=2}^{m}\left(\frac{c+1}{c+n}\right) a_{n} z^{n}, \quad(z \in U)
$$

and

$$
F_{m}(z)=z+\sum_{n=2}^{m} \frac{a_{n}}{[1+(n-1) \lambda]^{k} C(\delta, n)} z^{n}, \quad(z \in U) .
$$

It was shown that for a normalized univalent function $f$ of the form (1) the partial sums of the Libera integral operator of functions is starlike in $|z|<\frac{3}{8}$. The number $\frac{3}{8}$ is sharp ([13]). In [14], it was also shown that the partial sums of the Libera integral operator of functions of bounded turning are also of bounded turning. We determine conditions under which the partial sums (5-7) of the multiplier integral operators (2-4) of analytic univalent functions of bounded turning are also of bounded turning. In the sequel we need to the following results.

Lemma 1.1 [14]. For $z \in U$ we have

$$
\Re\left\{\sum_{n=1}^{j} \frac{z^{n}}{n+2}\right\}>-\frac{1}{3}, \quad(z \in U) .
$$

Lemma $1.2[1$, Vol. I]. Let $P(z)$ be analytic in $U$, such that $P(0)=1$, and $\Re(P(z))>\frac{1}{2}$ in $U$. For functions $Q$ analytic in $U$ the convolution function $P * Q$ takes values in the convex hull of the image on $U$ under $Q$.

The operator $(*)$ stands for the Hadamard product or convolution of two power series in $\mathcal{A}$,

$$
f(z) * g(z)=z+\sum_{n=2}^{\infty} a_{n} b_{n} z^{n}, \quad(z \in U) .
$$

\section{Main Results}

By making use of Lemma 1.1 and Lemma 1.2, we illustrate the conditions under which the $m$-th partial sums (5-7) of the multiplier integral operators (2-4) of analytic univalent functions of bounded turning are also of bounded turning. 
Theorem 2.1. Let $f \in \mathcal{A}$. If $\frac{1}{2}<\mu<1$ and $f(z) \in B(\mu)$, then

$$
P_{m}(z) \in B\left(\frac{3-(b+1)^{a}(1-\mu)}{3}\right), 0<a \leq 1 \text { and }-1<b \leq 1 .
$$

Proof. Let $f$ be of the form (1) and $f(z) \in B(\mu)$ that is

$$
\Re\left\{f^{\prime}(z)\right\}>\mu, \quad\left(\frac{1}{2}<\mu<1, \quad z \in U\right) .
$$

This implies

$$
\Re\left\{1+\sum_{n=2}^{\infty} n a_{n} z^{n-1}\right\}>\mu>\frac{1}{2} .
$$

Now for $\frac{1}{2}<\mu<1$ we have

$$
\Re\left\{1+\sum_{n=2}^{\infty} a_{n} \frac{n}{1-\mu} z^{n-1}\right\}>\Re\left\{1+\sum_{n=2}^{\infty} n a_{n} z^{n-1}\right\}
$$

then

$$
\Re\left\{1+\sum_{n=2}^{\infty} \frac{n}{1-\mu} a_{n} z^{n-1}\right\}>\frac{1}{2} .
$$

Applying the convolution properties of power series to $P_{m}^{\prime}(z)$ we may write

$$
\begin{aligned}
P_{m}^{\prime}(z) & =1+\sum_{n=2}^{m}\left(\frac{b+1}{b+n}\right)^{a} n a_{n} z^{n-1} \\
& =\left[1+\sum_{n=2}^{m} \frac{n}{(1-\mu)} a_{n} z^{n-1}\right] *\left[1+\sum_{n=2}^{m}\left(\frac{b+1}{b+n}\right)^{a}(1-\mu) z^{n-1}\right] \\
& :=P(z) * Q(z) .
\end{aligned}
$$

In virtue of Lemma 1.1 and for $j=m-1$, we receive

$$
\Re\left\{\sum_{n=2}^{m} \frac{z^{n-1}}{n+1}\right\}>-\frac{1}{3} .
$$

Thus for $0<a \leq 1$ and $-1<b \leq 1$ yields

$$
\Re\left\{\sum_{n=2}^{m} \frac{z^{n-1}}{(b+n)^{a}}\right\} \geq \Re\left\{\sum_{n=2}^{m} \frac{z^{n-1}}{n+1}\right\} .
$$


Hence

$$
\Re\left\{\sum_{n=2}^{m} \frac{z^{n-1}}{(b+n)^{a}}\right\}>-\frac{1}{3} .
$$

A computation gives

$$
\Re\{Q(z)\}=\Re\left\{1+\sum_{n=2}^{m}\left(\frac{b+1}{b+n}\right)^{a}(1-\mu) z^{n-1}\right\}>\frac{3-(b+1)^{a}(1-\mu)}{3} .
$$

On the other hand, the power series

$$
P(z)=\left[1+\sum_{n=2}^{m} \frac{n}{(1-\mu)} a_{n} z^{n-1}\right], \quad(z \in U)
$$

satisfies: $P(0)=1$ and

$$
\Re\{P(z)\}=\Re\left\{1+\sum_{n=2}^{m} \frac{n}{(1-\mu)} a_{n} z^{n-1}\right\}>\frac{1}{2}, \quad(z \in U) .
$$

Therefore, by Lemma 1.2, we have

$$
\Re\left\{P_{m}^{\prime}(z)\right\}>\frac{3-(b+1)^{a}(1-\mu)}{3}, \quad(z \in U) .
$$

This completes the proof of Theorem 2.1.

In the next corollary, we establish the conditions of the partial sums of the operator (3) to be of bounded turning when $f$ is of bounded turning.

Corollary 2.1. Let $f \in \mathcal{A}$. If $\frac{1}{2}<\mu<1$ and $f(z) \in B(\mu)$, then $J_{m}(z) \in$ $B\left(\frac{3-(c+1)(1-\mu)}{3}\right)$.

Proof. Setting $a=1$ and $b=c$ in Theorem 2.1 leads to Corollary 2.1.

Corollary 2.2. Let $f \in \mathcal{A}$. If $\frac{1}{2}<\mu<1$ and $f(z) \in B(\mu)$, then $L_{m}(z) \in$ $B\left(\frac{1+2 \mu}{3}\right)$, where $L(z)$ denotes the Libera integral operator:

$$
L(z)=\frac{2}{z} \int_{0}^{z} f(\zeta) d \zeta=z+\sum_{n=2}^{\infty}\left[\frac{2}{n+1}\right] a_{n} z^{n}, \quad(z \in U)
$$


and its $m$-th partial sums are given by

$$
L_{m}(z)=z+\sum_{n=2}^{m}\left[\frac{2}{n+1}\right] a_{n} z^{n}, \quad(z \in U) .
$$

Proof. Setting $a=b=1$ in Theorem 2.1 leads to Corollary 2.2.

Corollary 2.3. Let $f \in \mathcal{A}$. If $\frac{1}{2}<\mu<1$ and $f(z) \in B(\mu)$, then $S_{m}(z) \in$ $B\left(\frac{2+\mu}{3}\right)$, where $S^{k}(z)$ denotes the integral operator which analogous to one defined by Sălăgean (see [15]):

$$
S^{k}(z)=z+\sum_{n=2}^{\infty} \frac{a_{n}}{n^{k}} z^{n}, \quad k \in \mathbb{N}_{0}, \quad(z \in U)
$$

and its $m$-th partial sums are given by

$$
S_{m}(z)=z+\sum_{n=2}^{m} \frac{a_{n}}{n^{k}} z^{n}, \quad k \in \mathbb{N}_{0}, \quad(z \in U) .
$$

Proof. Setting $a=k, b=0$ in Theorem 2.1 leads to Corollary 2.3.

Theorem 2.2. Let $f \in \mathcal{A}$. If $\frac{1}{2}<\mu<1$ and $f(z) \in B(\mu)$, then

$$
F_{m}(z) \in B\left(\frac{2+\mu}{3}\right), \quad \delta=0, k=1, \text { and } 0 \leq \lambda \leq 1 \text {. }
$$

Proof. By the hypotheses of the theorem we have

$$
\Re\left\{1+\sum_{n=2}^{\infty} n a_{n} z^{n-1}\right\}>\mu>\frac{1}{2} .
$$

This implies, for $\frac{1}{2}<\mu<1$,

$$
\Re\left\{1+\sum_{n=2}^{\infty} a_{n} \frac{n}{1-\mu} z^{n-1}\right\}>\Re\left\{1+\sum_{n=2}^{\infty} n a_{n} z^{n-1}\right\}
$$

then

$$
\Re\left\{1+\sum_{n=2}^{\infty} \frac{n}{1-\mu} a_{n} z^{n-1}\right\}>\frac{1}{2} .
$$


Applying the convolution properties of power series to $F_{m}^{\prime}(z)$ we have

$$
\begin{aligned}
F_{m}^{\prime}(z)= & 1+\sum_{n=2}^{m} \frac{n a_{n}}{[1+(n-1) \lambda]^{k} C(\delta, n)} z^{n-1} \\
= & {\left[1+\sum_{n=2}^{m} \frac{n a_{n}}{(1-\mu)} z^{n-1}\right] } \\
& \times\left[1+\sum_{n=2}^{m} \frac{(1-\mu)}{[1+(n-1) \lambda]^{k} C(\delta, n)} z^{n-1}\right] \\
:= & P(z) * Q(z) .
\end{aligned}
$$

In view of Lemma 1.1 with

$$
j=m-1, \quad \delta=0, \quad k=1, \quad \text { and } \quad 0 \leq \lambda \leq 1,
$$

yields

$$
\Re\left\{\sum_{n=2}^{m} \frac{z^{n-1}}{[1+(n-1) \lambda]}\right\}>\Re\left\{\sum_{n=2}^{m} \frac{z^{n-1}}{n+1}\right\} .
$$

Hence

$$
\Re\left\{\sum_{n=2}^{m} \frac{z^{n-1}}{[1+(n-1) \lambda]}\right\}>-\frac{1}{3} .
$$

Under the conditions given in (16) we obtain

$$
\Re\{Q(z)\}=\Re\left\{1+\sum_{n=2}^{m} \frac{(1-\mu)}{[1+(n-1) \lambda]} z^{n-1}\right\}>\frac{2+\mu}{3} .
$$

On the other hand, the power series

$$
P(z)=\left[1+\sum_{n=2}^{m} \frac{n}{(1-\mu)} a_{n} z^{n-1}\right], \quad(z \in U)
$$

satisfies: $P(0)=1$ and

$$
\Re\{P(z)\}=\Re\left\{1+\sum_{n=2}^{m} \frac{n}{(1-\mu)} a_{n} z^{n-1}\right\}>\frac{1}{2}, \quad(z \in U) .
$$

Therefore, by Lemma 1.2, we have

$$
\Re\left\{P_{m}^{\prime}(z)\right\}>\frac{2+\mu}{3},\left(\frac{1}{2}<\mu<1\right) .
$$

The proof of Theorem 2.2 is complete. 
Corollary 2.4. Let $f \in \mathcal{A}$. If $\frac{1}{2}<\mu<1$ and $f(z) \in B(\mu)$, then $S_{m}(z) \in$ $B\left(\frac{2+\mu}{3}\right)$, where $S(z)$ defined in (13) of order one.

Proof. Setting $\lambda=1$ in Theorem 2.2 leads to Corollary 2.4.

Acknowledgement. The authors were supported in part by ScienceFund: 04-01-02-SF0425, MOSTI, Malaysia.

\section{REFERENCES}

[1] A.W. Goodman, Univalent Functions, Vols. I and II, Polygonal Publishing House, Washington, New Jersey (1983).

[2] S. Owa and H.M. Srivastava, Some applications of the generalized Libera integral operator. Proc. Japan Acad. Ser. A Math. Sc., 62 (1986), 125-128.

[3] H.M. Srivastava and S. Owa, New characterizations of certain starlike and convex generahzed hypergeometric functions. J. Nat. Acad Math. India, 3 (1985), 198-202.

[4] H.M. Srivastava and S. Owa, A certain one-parameter additive family of operators defined on analytic functions. J. Math. Anal. Appl., 118 (1986), 80-87.

[5] J -L. Liu, On a class of p-valent analytic functions. Chinese Quart. J. Math., 15(4) (2000), $27-32$.

[6] J.-L. Liu, Some applications of certain integral operator. Kyungpook Math. J., 43 (2003), 211-219.

[7] J.-L Liu, Notes on Jung-Kim-Snvastava integral operator. J. Math. Anal. Appl., 294 (2004), 96-103.

[8] B.A. Uralegaddi and C. Somanatha, Certain integral operators for starlike functions. J. Math Res. Exposition, 14-16 (1995).

[9] J-L Liu, Some properties of two integral operators. Soochow J. Math., 25 (1999), 91-96.

[10] S. Owa, Properties of certain integral operators. Georgian Math. J., 2 (1995), 535-545.

[11] K.I. Noor, On new classes of integral operators. J. Nat. Geomet., 16 (1999), 71-80.

[12] K.I. Noor and M.A. Noor, On integral operators. J. Math. Anal. Appl., 238 (1999), 341-352.

[13] J.L. Liu and S. Owa, On partial sums of the Libera integral operator. J. Math. Anal. Appl., 213 (1997), 444-454.

[14] J.M. Jahangiri, K. Farahmand, Partial sums of functions of bounded turning. Inter. J. Math. Math. Sci., 2004(1), (2004), 45-47.

[15] G.S. Sălăgean, Subclasses of univalent functions. Lecture Notes in Math., 1013 (1983), 362-372. Springer-Verlag, Berlin. 